\title{
On the Full Dimensionality Assumption for the Discounted Folk Theorem*
}

\author{
Guilherme Carmona ${ }^{\dagger}$ \\ Universidade Nova de Lisboa
}

November 12, 2008

\begin{abstract}
A sufficient condition for the discounted Folk Theorem is that for every individually rational payoff vector $u$, there exists another individually rational payoff vector $v$ such that $v<u$. We present an example of a full dimensional game where this property fails to be satisfied. Furthermore, we present a simple and direct proof that full dimensionality implies a weaker, but still sufficient property for the discounted Folk Theorem.
\end{abstract}

Journal of Economic Literature Classification Numbers: C73

Keywords: Folk theorem, full dimensionality

\section{Introduction}

The discounted Folk Theorem states that, under some appropriate condition, every individual rational payoff vector of a normal-form game can be supported by a subgame perfect equilibrium strategy of the discounted repeated games it induces.

${ }^{*}$ I thank Hamid Sabourian for several comments and John Huffstot for editorial assistance. Financial support from a Nova Fórum grant is gratefully acknowledged.

${ }^{\dagger}$ Address: Universidade Nova de Lisboa, Faculdade de Economia, Campus de Campolide, 1099032 Lisboa, Portugal; Phone: (351) 21380 1672; Fax: (351) 21387 0933; email: gcarmona@fe.unl.pt. 
The most widely used sufficient condition for the discounted Folk Theorem was introduced by Fudenberg and Maskin (1986) and requires the set of individually rational payoffs to be full dimensional. A close reading of the proof of FudenbergMaskin's Theorem 2 reveals, however, that all one needs to establish it is to be able to slightly perturb the payoff we want to support inside the set of individually rational payoffs.

Two types of perturbations have, in fact, been considered in that proof: the first, which we shall refer to as the strong Fudenberg-Maskin property, requires that for every individually rational payoff $u$ there exists another individually rational payoff $v$ satisfying $v<u$. The second, which we name the Fudenberg-Maskin property, requires only that for every individually rational payoff $u$ there exist individually rational payoff vectors $\left\{u^{(1)}, \ldots, u^{(n)}\right\}$, one for every player $i=1, \ldots, n$, satisfying $u_{i}^{(i)}<u_{i}$ for all $i$ and the payoff asymmetry condition in Abreu, Dutta, and Smith (1994).

The main goal of this note is to present an example showing that the full dimensionality assumption does not imply the strong Fudenberg-Maskin property. Our example confirms the intuition expressed in Abreu, Dutta, and Smith (1994, Footnote 7) that the construction required by the strong Fudenberg-Maskin property may not be possible whenever the payoff $u$ lies on the lower boundary of the set of individually rational payoffs.

A second goal of this note is to provide a direct proof of the fact that the full dimensionality assumption implies the Fudenberg-Maskin property. Although this result can be established using the methods of Abreu, Dutta, and Smith (1994) (which also have the advantage of reaching the same conclusion with a weaker condition), the proof presented here has some interest by being simpler, shorter, and of course, by linking directly the full dimensionality assumption and the Fudenberg-Maskin property. 


\section{Notation and Definitions}

As in Fudenberg and Maskin (1986), we consider a $n$-person game $g: A_{1} \times \cdots \times A_{n} \rightarrow$ $\mathbb{R}^{n}$. For convenience, let $N=\{1, \ldots, n\}$ denote the set of players, $A=A_{1} \times \cdots \times A_{n}$ denote the joint action space and $A_{-i}=\prod_{j \neq i} A_{j}$.

For all $i \in N$, the minmax payoff for player $i$ is

$$
v_{i}^{*}=\min _{a_{-i} \in A_{-i}} \max _{a_{i} \in A_{i}} g_{i}\left(a_{i}, a_{-i}\right)
$$

and let $M^{i}$ be a minmax strategy against player $i$, i.e.,

$$
M^{i} \in \arg \min _{a_{-i} \in A_{-i}} \max _{a_{i} \in A_{i}} g_{i}\left(a_{i}, a_{-i}\right) .
$$

The vector $v^{*}=\left(v_{1}^{*}, \ldots, v_{n}^{*}\right)$ is the minmax point, and we normalize the payoffs of the game $g$ so that $v^{*}=0$. Let $V=\operatorname{co}(g(A))$ denote the set of feasible payoffs and $V^{*}=\left\{v \in V: v_{i}>0\right.$ for all $\left.i \in N\right\}$ denote the set of individually rational payoffs.

A game $g$ has the strong Fudenberg-Maskin property if for all $u \in V^{*}$ there exists $v \in \operatorname{int}\left(V^{*}\right)$ such that $v<u$. A game $g$ has the Fudenberg-Maskin property if for all $u \in V^{*}$ and $i \in N$ there exists $u^{(i)} \in V^{*}$ satisfying: $u_{i}^{(i)}<u_{i}$ and $u_{i}^{(i)}<u_{i}^{(j)}$ for all $i, j \in N$ with $j \neq i$. As was shown by Fudenberg and Maskin (1986) in the course of the proof of their Theorem 2, if $u \in V^{*}$ has the strong $F M$ property, then $u$ has the Fudenberg-Maskin property.

We shall use the following standard notation: $\left\{e_{1}, \ldots, e_{n}\right\}$ denotes the standard basis of $\mathbb{R}^{n}$, for all $u \in \mathbb{R}^{n},\|u\|$ denotes the sup norm of $u$, and for all $\varepsilon>0$ and $x \in \mathbb{R}, B_{\varepsilon}(x)$ denotes the open ball of radius $\varepsilon$ around $x$.

\section{$3 \quad$ Full Dimensionality and the Fudenberg-Maskin Property}

Since $V^{*}$ is a convex subset of $\mathbb{R}^{n}$, we can meaningfully speak of its dimension (see Rockafellar (1970, p. 12)). In general, let $C$ be a nonempty convex subset of $\mathbb{R}^{m}$. The affine hull, aff $(C)$, of $C$ is the smallest affine set containing $S .{ }^{1}$ By Rockafellar

\footnotetext{
${ }^{1}$ Recall that a subset $M$ of $\mathbb{R}^{m}$ is an affine set if $(1-\lambda) x+\lambda y \in M$ for every $x, y \in M$ and $\lambda \in \mathbb{R}$.
} 
(1970, Theorem 1.2), each nonempty affine set is parallel to a unique subspace, i.e., there exists $a \in \mathbb{R}^{m}$ and a unique subspace $L$ of $\mathbb{R}^{m}$ such that $M=L+a$. Then, the dimension, $\operatorname{dim}(C)$, of $C$ is the dimension of the subspace parallel to its affine hull.

A convex subset $C$ of $\mathbb{R}^{m}$ is full dimensional if $\operatorname{dim}(C)=m$. Note that this condition holds if and only if $\operatorname{int}(C)$ is nonempty. In fact, the only if part follows from Rockafellar (1970, Theorem 6.2, p.45), while the if part can be established as follows: if $x \in \operatorname{int}(C)$ and $\varepsilon>0$ is such that $B_{2 \varepsilon}(x) \subseteq C$, then $\left\{x, x+\varepsilon e_{1}, \ldots, x+\varepsilon e_{m}\right\} \subseteq C$ is affinely independent, and so aff $\left(\left\{x, x+\varepsilon e_{1}, \ldots, x+\varepsilon e_{m}\right\}\right)$ is full dimensional. Hence, $\operatorname{dim}(C)=\operatorname{dim}(\operatorname{aff}(C))=m$.

The following example shows that a game may fail to have the strong FudenbergMaskin property even if $V^{*}$ is full dimensional.

Consider the following three-player game where player 1 chooses a row $(a$ or $b)$, player 2 a column $(A$ or $B)$ and player 3 a matrix $(\alpha$ or $\beta)$. If player 3 chooses $\alpha$, then payoffs are:

\begin{tabular}{|c|c|c|}
\hline & $A$ & $B$ \\
\hline$a$ & $(0,1,0)$ & $(1,0,0)$ \\
$b$ & $(0,1,0)$ & $(1,0,0)$ \\
\hline
\end{tabular}

while if player 3 chooses $\beta$, payoffs are:

\begin{tabular}{|c|c|c|}
\hline & $A$ & $B$ \\
\hline$a$ & $(0,0,1)$ & $(0,0,1)$ \\
$b$ & $(1,1,0)$ & $(1,0,0)$ \\
\hline
\end{tabular}

Note that player 1 receives 0 if the others play $(A, \alpha)$, player 2 receives 0 if the others play $(a, \beta)$ and player 3 receives 0 if the others play $(b, B)$. Hence $v_{i}^{*}=0$ for all $i=1,2,3$.

The equivalence between the full dimensionality of $V^{*}$ and the nonemptiness of its interior imply easily that if $\breve{V}=\left\{v \in V: v_{i} \geq 0\right\}$ is full dimensional, then so is $V^{*}$ since $\operatorname{int}(\breve{V})=\operatorname{int}\left(V^{*}\right)$.

Hence, in order to prove that $V^{*}$ is full dimensional, it is enough to show that $\operatorname{dim}(\breve{V})=3$, which is equivalent to $\operatorname{aff}(\breve{V})=\mathbb{R}^{3}$. Letting $c_{1}=(1,0,0)=e_{1}, c_{2}=$ 
$(0,1,0)=e_{2}, c_{3}=(0,0,1)=e_{3}$ and $c_{4}=(1,1,0)$, then

$$
\begin{aligned}
& \operatorname{aff}(\breve{V})=\left\{\sum_{j=1}^{4} \lambda_{j} c_{j}: \sum_{j=1}^{4} \lambda_{j}=1\right\}=\left\{\left(\lambda_{1}+\lambda_{4}, \lambda_{2}+\lambda_{4}, \lambda_{3}\right): \sum_{j=1}^{4} \lambda_{j}=1\right\} \\
& =\left\{\left(1-\lambda_{2}-\lambda_{3}, 1-\lambda_{1}-\lambda_{3}, \lambda_{3}\right): \lambda_{1}, \lambda_{2}, \lambda_{3} \in \mathbb{R}\right\} .
\end{aligned}
$$

Hence, if $x=\left(x_{1}, x_{2}, x_{3}\right) \in \mathbb{R}^{3}$, then by defining $\lambda_{1}=1-x_{2}-x_{3}, \lambda_{2}=1-x_{1}-x_{3}$, $\lambda_{3}=x_{3}$ and $\lambda_{4}=1-\sum_{j=1}^{3} \lambda_{j}$, it follows that $x=\sum_{j=1}^{4} \lambda_{j} c_{j} \in \operatorname{aff}(\breve{V})$. Thus, $\operatorname{aff}(\breve{V})=\mathbb{R}^{3}$.

Note that, for all $u \in V^{*} \subseteq V=\operatorname{co}(g(A))$, we have that $u=\sum_{j=1}^{4} \theta_{j} c_{j}$ for some $\left\{\theta_{j}\right\}_{j=1}^{4}$ such that $\theta_{j} \geq 0$ for all $j$ and $\sum_{j} \theta_{j}=1$. Hence, $\sum_{i=1}^{3} u_{i}=1+\theta_{4} \geq 1$.

Consider $u=(1 / 3,1 / 3,1 / 3) \in V^{*}$. Suppose, in order to reach a contradiction, that there exists $v \in \operatorname{int}\left(V^{*}\right)$ such that $v<u$. Then, $\sum_{i=1}^{3} v_{i}<1$. However, since $v \in V^{*}$, then it follows by the above that $\sum_{i=1}^{3} v_{i} \geq 1$, a clear contradiction. Thus, this game does not satisfy the strong Fudenberg-Maskin property.

Although the full dimensionality of $V^{*}$ does not imply the strong FudenbergMaskin property, it suffices to establish the Fudenberg-Maskin property.

Theorem 1 If $\operatorname{dim}\left(V^{*}\right)=n$, then $g$ has the Fudenberg-Maskin property.

Proof. Let $u \in V^{*}$. Since $\operatorname{dim}\left(V^{*}\right)=n$, then $\operatorname{int}\left(V^{*}\right)$ is nonempty and so let $u_{0} \in \operatorname{int}\left(V^{*}\right)$. For all $i \in N$, define

$$
\tilde{u}^{(i)}=\lambda\left(\theta u+(1-\theta) g\left(M^{i}\right)\right)+(1-\lambda) u_{0}
$$

where $\theta \in(0,1)$ is chosen sufficiently close to 1 so that $\theta u+(1-\theta) g\left(M^{i}\right) \in V^{*}$ and $\lambda \in(0,1)$ is chosen sufficiently close to 1 so that $\tilde{u}_{i}^{(i)}<u_{i}$ (note that the payoff for player $i$ in $\theta u+(1-\theta) g\left(M^{i}\right)$ is equal to $\left.\theta u_{i}<u_{i}\right)$. Since $\theta u+(1-\theta) g\left(M^{i}\right)$ belongs to $V^{*}, u_{0} \in \operatorname{int}\left(V^{*}\right)$ and $\lambda \in(0,1)$, it follows from Rockafellar (1970, Theorem 6.1, p.

45) that $\tilde{u}^{(i)} \in \operatorname{int}\left(V^{*}\right)$. Let $\varepsilon>0$ be such that $B_{2 \varepsilon}\left(\tilde{u}^{(i)}\right) \subseteq V^{*}$ for all $i \in N$.

Let $i \in N$ and let $k(i) \in N$ be such that $\tilde{u}_{i}^{(k(i))} \leq \tilde{u}_{i}^{(l)}$ for all $l \in N$. Then, define

$$
u^{(i)}=\tilde{u}^{(k(i))}-\varepsilon e_{i}
$$


Since $\left\|u^{(i)}-\tilde{u}^{(k(i))}\right\|=\varepsilon$, it follows that $u^{(i)} \in V^{*}$ for all $i \in N$. Furthermore, we have that $u_{i}^{(i)}<u_{i}$ for all $i \in N$. Indeed, $u_{i}^{(i)}=\tilde{u}_{i}^{(k(i))}-\varepsilon \leq \tilde{u}_{i}^{(i)}-\varepsilon<u_{i}$. Finally, we claim that $u_{i}^{(i)}<u_{i}^{(j)}$ for all $i, j \in N$ such that $j \neq i$. Indeed,

$$
u_{i}^{(i)}=\tilde{u}_{i}^{(k(i))}-\varepsilon \leq \tilde{u}_{i}^{(k(j))}-\varepsilon=u_{i}^{(j)}-\varepsilon<u_{i}^{(j)} .
$$

Hence, $u$ satisfies the Fudenberg-Maskin property.

\section{References}

Abreu, D., P. Dutta, And L. Smith (1994): "The Folk Theorem for Repeated Games: A Neu Condition," Econometrica, 62, 939-948.

Fudenberg, D., And E. Maskin (1986): "The Folk Theorem in Repeated Games with Discounting or with Incomplete Information," Econometrica, 54, 533-554.

Rockafellar, R. T. (1970): Convex Analysis. Princeton University Press, Princeton. 